ARTICLE

Received 4 Sep 2013 | Accepted 11 Oct 2013 | Published 15 Nov $2013 \quad$ DOl: 10.1038/ncomms3750

\title{
Transition fibre protein FBF1 is required for the ciliary entry of assembled intraflagellar transport complexes
}

Qing Wei ${ }^{1}$, Qingwen Xu², Yuxia Zhang ${ }^{1}$, Yujie $\mathrm{Li}^{1}$, Qing Zhang ${ }^{1}$, Zeng $\mathrm{Hu}^{1}$, Peter C. Harris ${ }^{1,3}$, Vicente E. Torres ${ }^{1,3}$, Kun Ling ${ }^{2,3}$ \& Jinghua $\mathrm{Hu}^{1,2,3}$

Sensory organelle cilia have critical roles in mammalian embryonic development and tissue homeostasis. Intraflagellar transport (IFT) machinery is required for the assembly and maintenance of cilia. Yet, how this large complex passes through the size-dependent barrier at the ciliary base remains enigmatic. Here we report that FBF1, a highly conserved transition fibre protein, is required for the ciliary import of assembled IFT particles at the ciliary base. We cloned dyf-19, the Caenorhabditis elegans homologue of human FBF1, in a whole-genome screen for ciliogenesis mutants. DYF-19 localizes specifically to transition fibres and interacts directly with the IFT-B component DYF-11/IFT54. Although not a structural component of transition fibres, DYF-19 is required for the transit of assembled IFT particles through the ciliary base. Furthermore, we found that human FBF1 shares conserved localization and function with its worm counterpart. We conclude that FBF1 is a key functional transition fibre component that actively facilitates the ciliary entry of assembled IFT machinery.

\footnotetext{
${ }^{1}$ Division of Nephrology and Hypertension, Mayo Clinic, Rochester, Minnesota 55905, USA. ${ }^{2}$ Department of Biochemistry and Molecular Biology, Mayo Clinic, Rochester, Minnesota 55905, USA. ${ }^{3}$ Mayo Translational PKD Center, Rochester, Minnesota 55905, USA. Correspondence and requests for materials should be addressed to K.L. (email: ling.kun@mayo.edu) or to J.H. (email: hu.jinghua@mayo.edu).
} 
$\mathrm{P}$ rimary cilia exist on most eukaryotic cell surfaces and function as the cell's antenna to transduce many critical cellular signalling pathways ${ }^{1,2}$. Cilia dysfunction has been linked to a wide spectrum of human genetic diseases, collectively known as ciliopathies ${ }^{3,4}$. Consistent with the ubiquitous presence of cilia on the surfaces of most cells in the human body, most ciliopathies occur as syndromic disorders that affect the homeostasis of many organs during development, including the kidneys, liver, limbs, eyes, central nervous system and fat storage tissue.

Intraflagellar transport (IFT) complexes are an evolutionarily conserved machinery that move bidirectionally from the ciliary base to the tip to transport cargos required for ciliogenesis and signalling $^{5-8}$. The IFT machinery comprises $>20$ proteins, including motors, IFT-A subcomplexes, IFT-B subcomplexes and the BBSome $\mathrm{e}^{5-8}$. Many structural components of the IFT machinery are large proteins with size $>60 \mathrm{kDa}$. In addition, some IFT cargoes, such as radial spokes and dynein arms, are also pre-assembled in the cytoplasm before their ciliary entry ${ }^{9,10}$. How these large proteins and/or complexes actively pass through the recently reported size-dependent barrier at the ciliary base remains poorly understood ${ }^{11,12}$.

Ultrastructural studies have shown that the morphology of the ciliary base is highly conserved, including a basal body (transformed from the mother centriole), transition fibres (TFs; transformed from the distal appendages of the mother centriole) and the transition zone (TZ; the proximal part of the axoneme that contains Y-links) ${ }^{13}$. TFs form a nine-bladed propeller-like structure of unknown protein composition and connect the distal end of the basal body to the ciliary membrane ${ }^{14}$. Above TFs, the Y-links of the TZ connect the proximal segments of axonemal microtubules to the ciliary membrane ${ }^{15}$. Recently, emerging evidence suggests that many proteins mutated in human ciliopathy syndromes specifically localize around the $\mathrm{TZ}$ and probably form a multimeric protein complex to regulate the integrity of Y-links and/or the ciliary entry of some ciliary proteins ${ }^{16-22}$. In drastic contrast, little is known about the molecular identity and function of TFs in the context of cilia.

The observations that IFT components concentrate around TFs in the preciliary compartment led to the hypothesis that TFs might be a critical site for the docking or sorting of IFT particles or individual components before they enter the cilium ${ }^{23}$. However, none of the identified distal appendage proteins (DAPs) have been shown to regulate the ciliary import or sorting of IFT particles or individual components ${ }^{24-27}$. In a whole-genome genetic screen for Caenorhabditis elegans mutants with disrupted IFT integrity, we retrieved and cloned $d y f-19$, the homologue of human FBF1. Both DYF-19 and FBF1 (Fas (TNFRSF6)-binding factor 1) are poorly characterized proteins. DYF-19 specifically localizes to TFs and is essential for the ciliary entry of IFT complexes through direct interaction with IFT-B component DYF-11 (the orthologue of human IFT54). DYF-19 deficiency does not affect TF assembly but results in the accumulation of assembled IFT particles below TFs. Furthermore, we found that human FBF1 is essential for ciliogenesis and shares conserved specific TF localization and function with its worm counterpart. Collectively, our findings demonstrate that FBF1 defines the TFs as the key site for regulating the ciliary entry of assembled IFT machinery.

\section{Results}

TF protein DYF-19 regulates ciliogenesis in C. elegans. To search for mutant nematodes with abnormal ciliogenesis, we performed a genome-wide ethyl methanesulphonate (EMS) mutagenesis screen in C. elegans. From this screen, we cloned a novel mutant, $d y f-19$. In C. elegans, mutants with abnormal ciliogenesis cannot take up fluorescent dye and are thus called dye-filling defective (Dyf ${ }^{28}$. $d y f-19$ encodes Y43F8C.4, which is the homologue of human FBF1 protein ${ }^{29}$ (Fig. 1a, Supplementary Fig. S1a). Originally identified as a keratin filament-binding protein $^{30}$, FBF1 shows asymmetric centrosome localization ${ }^{31}$ and was recently confirmed as one of the DAPs ${ }^{25}$, but with uncharacterized function. Our bioinformatics analyses indicate that FBF1 is the only DAP identified so far (CEP164, CEP89, CEP83, SCLT1 and FBF1 (ref. 25)) with a clear homologue in the worm genome. This suggests that FBF1 probably plays a highly conserved and important role on TFs. $d y f-19$ mutants possessed truncated cilia and exhibited abnormal accumulation of the IFT-B component OSM-6 (the orthologue of human IFT52) at the residual cilia tip (Fig. 1b,c). Few or no IFT movements were observed in $d y f-19$ cilia. Introducing a wild-type (wt) Y43F8C.4 gene fully rescued the ciliogenesis defect in $d y f-19$ mutants (Fig. 1c,d).

Promoter expression analysis demonstrated that $d y f-19$ is expressed exclusively in ciliated cells (Supplementary Fig. S1b). At the $C$. elegans ciliary base, the periciliary membrane compartment was found immediately below the $\mathrm{TFs}^{32}$, and the TZ was located just above the $\mathrm{TFs}^{13}$ (Fig. 1g). mCherry-tagged DYF-19 was found to localize above the periciliary membrane compartment marker green fluorescent protein (GFP)-tagged RPI-2 (the worm homologue of human X-linked retinitis pigmentosa 2), but below the TZ markers GFP-tagged NPHP-1 or MKS-5 in the cilia, indicating that DYF-19 is a genuine TF component (Fig. 1e-g). Truncated DYF-19 ${ }^{1-294}$, encoded by the $j h u 455$ allele used in our experiments, failed to target cilia and accumulated only in cell bodies (Fig. 1a, Supplementary Fig. S1c), suggesting that $d y f$ 19 (jhu455) is functionally null. The observation that dyf-19(jhu455) worms still possess normal TFs at the ciliary base indicates that DYF-19 is a functional, but not structural, component of TFs (Fig. 1h).

The function of DYF-19 is independent of the TZ. TFs and the $\mathrm{TZ}$ are spatially located adjacent to each other. The $\mathrm{TZ}$ is thought to restrict the ciliary entry of some non-ciliary proteins ${ }^{20}$. We observed no abnormality in either the localization of $\mathrm{TZ}$ proteins or the morphology of TZ Y-links in dyf-19 mutants (Supplementary Fig. S2a,b). It was reported that Meckel-Gruber (MKS) and nephronophthisis (NPHP) modules cooperate to establish a functional $\mathrm{TZ}^{20,33-35}$. In C. elegans, cilia exist at the tips of dendrites in ciliated sensory neurons. The combination of any $m k s$ mutant with an $n p h p$ mutant will disrupt TZ function and result in severely truncated dendrites due to perturbed anchoring of basal bodies ${ }^{20,33,34}$. Unlike $m k s-6$; $n p h p-1$ mutants, $d y f-19 ; m k s-6$ or $d y f-19 ; n p h p-1$ double mutants possessed wellformed dendrites (Supplementary Fig. S2c), indicating that $d y f-19$ does not genetically interact with $\mathrm{TZ}$ components.

DYF-19 is required for the ciliary entry of IFT particles. IFT is indispensable for ciliogenesis. To discern the role of DYF-19 and of TFs in IFT regulation, we examined various GFP-tagged IFT components in $d y f-19$ mutants. Like OSM-6 (Fig. 1c), other IFT-B components or the IFT-B-associated kinesin motor OSM-3 (the orthologue of human KIF17) accumulated at the tips of truncated $d y f-19$ cilia (Fig. 2a) and showed little or no IFT motility. In striking contrast, the IFT-A component CHE-11 (the orthologue of human IFT140), IFT-A-associated kinesin-II motor subunit KAP-1, IFT retrograde motor dynein light chain XBX-1 (the orthologue of human D2LIC) and BBSome components lost their ciliary presence in $d y f-19$ mutants (Fig. 2a). The ciliary absence of the IFT-A subcomplex and dynein (key factors in the retrograde IFT machinery ${ }^{36-41}$ ), and of the BBSome ${ }^{42,43}$ (key factors in IFT 


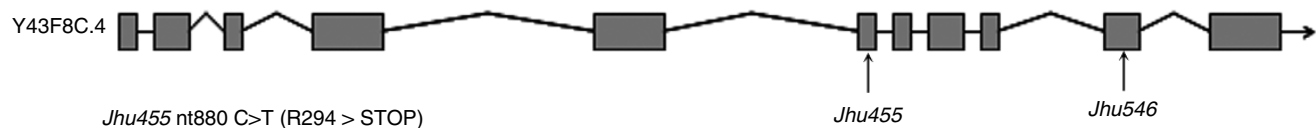

Jhu546 nt1219 C>T (Q407 > STOP)

b

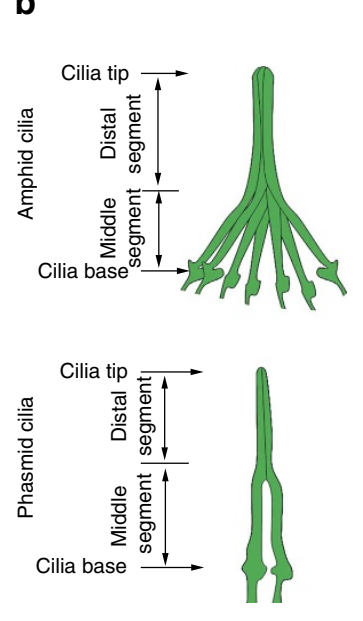

c

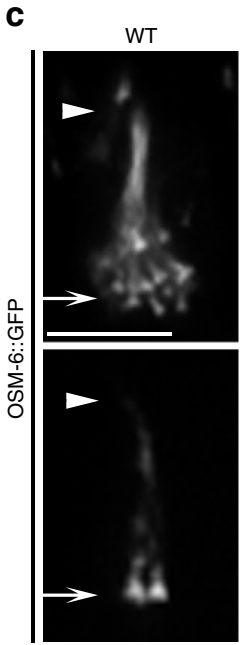

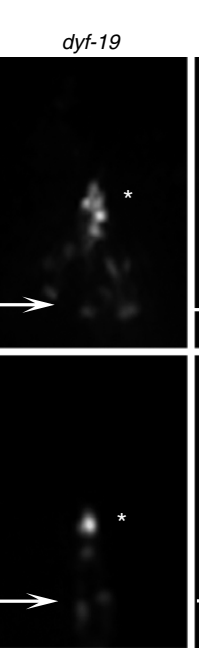

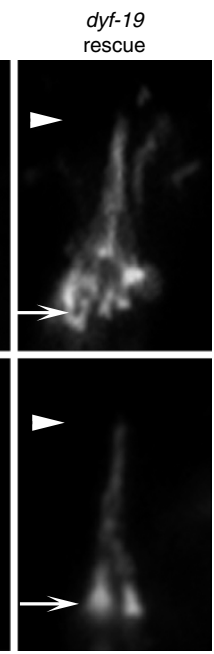

f

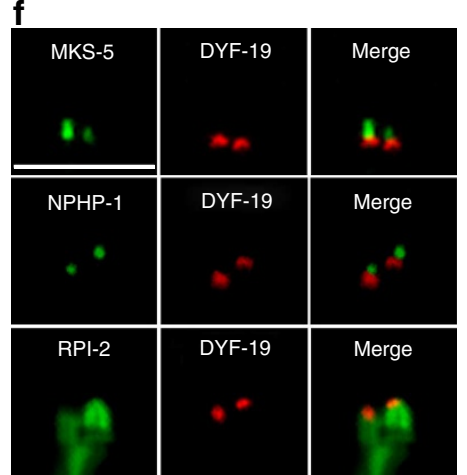

d

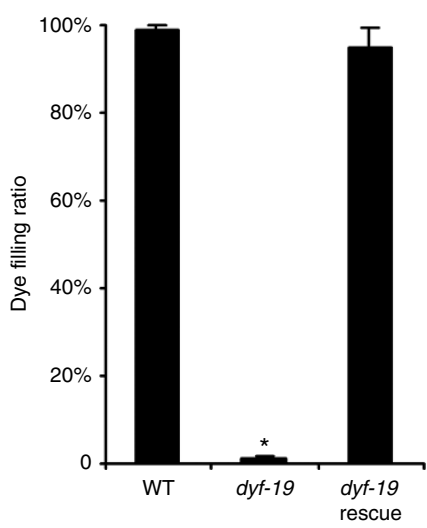

e

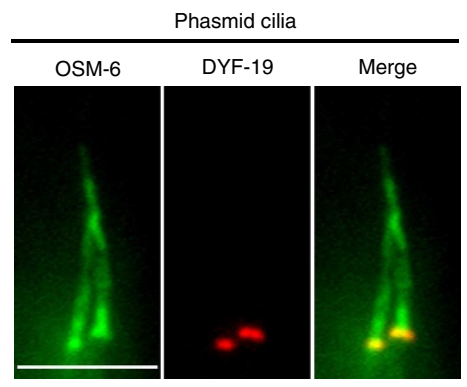

dyf-19

h WT

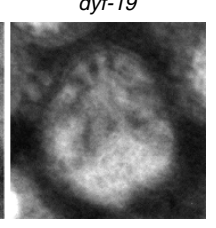

Figure 1 | DYF-19 is a functional component of TFs. (a) Schematic of Y43F8C.4 alleles. Jhu455 and jhu546 were retrieved from a genome-wide

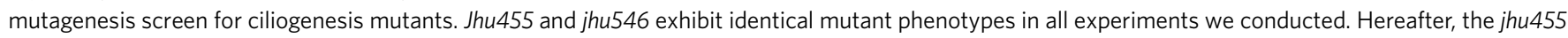

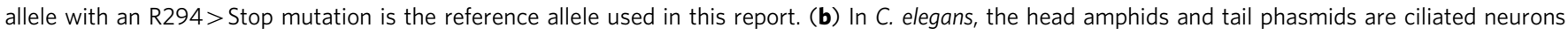
and primary sensory organs. The amphid cilia consist of a bundle of ten cilia and the phasmid has two cilia. (c) Fluorescence micrographs of cilia labelled with the GFP-tagged IFT-B component OSM-6 in Wt, dyf-19(jhu455) and dyf-19 rescue strain. dyf-19 shows truncated cilia and abnormal accumulation of OSM-6::GFP at the tips of residual cilia. Arrows and arrowheads indicate the base and tip of cilia, respectively. Stars note the accumulation of OSM-6::GFP. (d) The Dyf in dyf-19(jhu455) is fully rescued by introducing a wt copy of the Y43F8C.4 gene. Data are represented as mean of five independent experiments $(n=300)$ and error bars indicate s.d. Significant differences were identified by the Student's $t$-test. ${ }^{\star} P<0.001$. (e) $m C h e r r y-$ tagged DYF-19 specifically localizes at the ciliary base. (f) Spatial relationships between DYF-19 and TZ markers (MKS-5 and NPHP-1) and between DYF-19 and a periciliary membrane compartment (PCMC) marker (RPI-2). (g) Cartoon illustrating the TF localization of DYF-19 at the ciliary base.

(h) Transmission electron microscopy reveals that dyf-19 mutants possess normal TFs. Scale bars, $200 \mathrm{~nm}$ in $\mathbf{h}$ and $5 \mu \mathrm{m}$ in other micrographs.

assembly ${ }^{44-47}$ ), explains the ciliary accumulation of IFT-B components. We further found that IFT-A and BBSome proteins were restricted below the TZ in $d y f-19$ cilia, suggesting that their ability to pass through TFs depends on DYF-19 (Fig. 2b-d).
When colabelling cilia with GFP-tagged CHE-11 (IFT-A) and mCherry-tagged OSM-6 (IFT-B), we noticed that IFT-A and IFT-B still co-localize at the $d y f-19$ ciliary base (Fig. 3a). We thus hypothesized that assembled IFT complexes might still exist at the ciliary base but fail to enter the cilium in dyf-19 
a

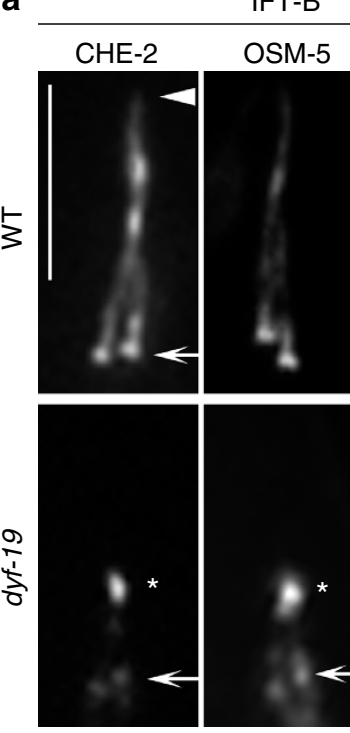

b
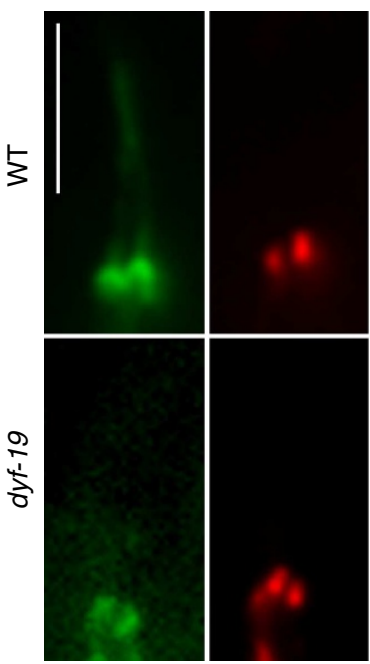

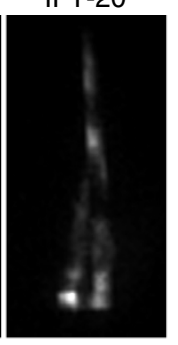

KIF17

OSM-3
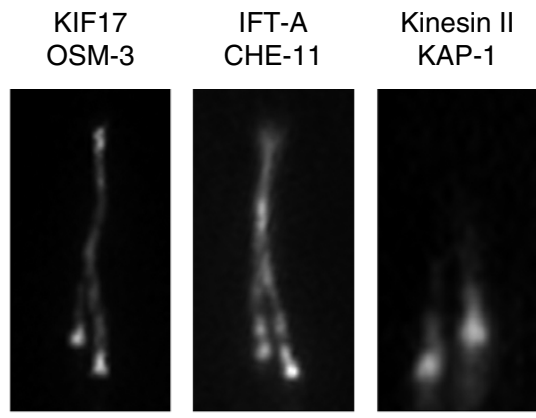

\section{Dynein \\ XBX-1}
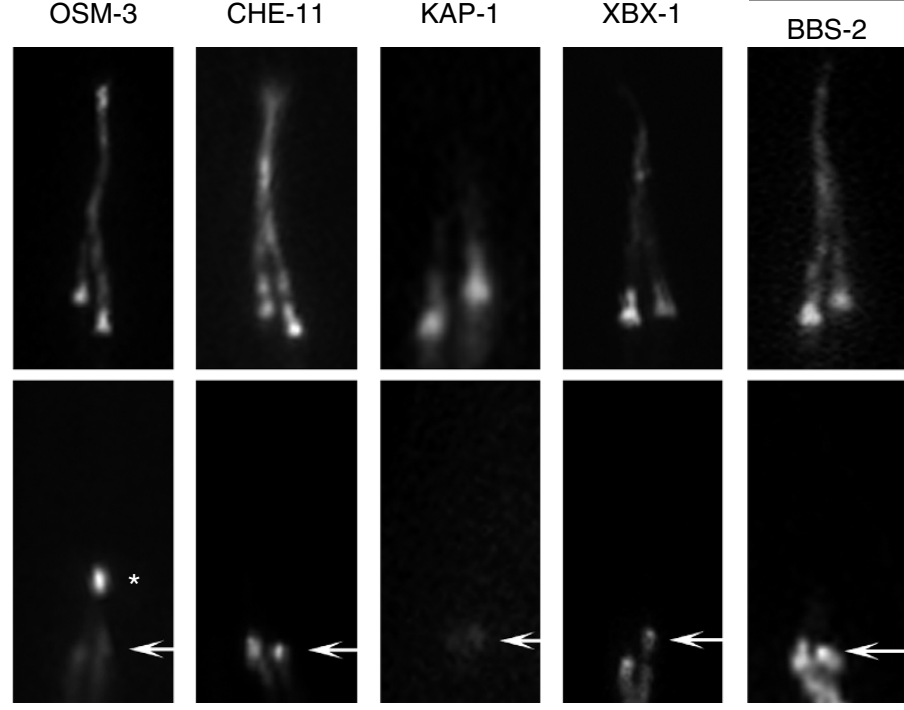

BBSome
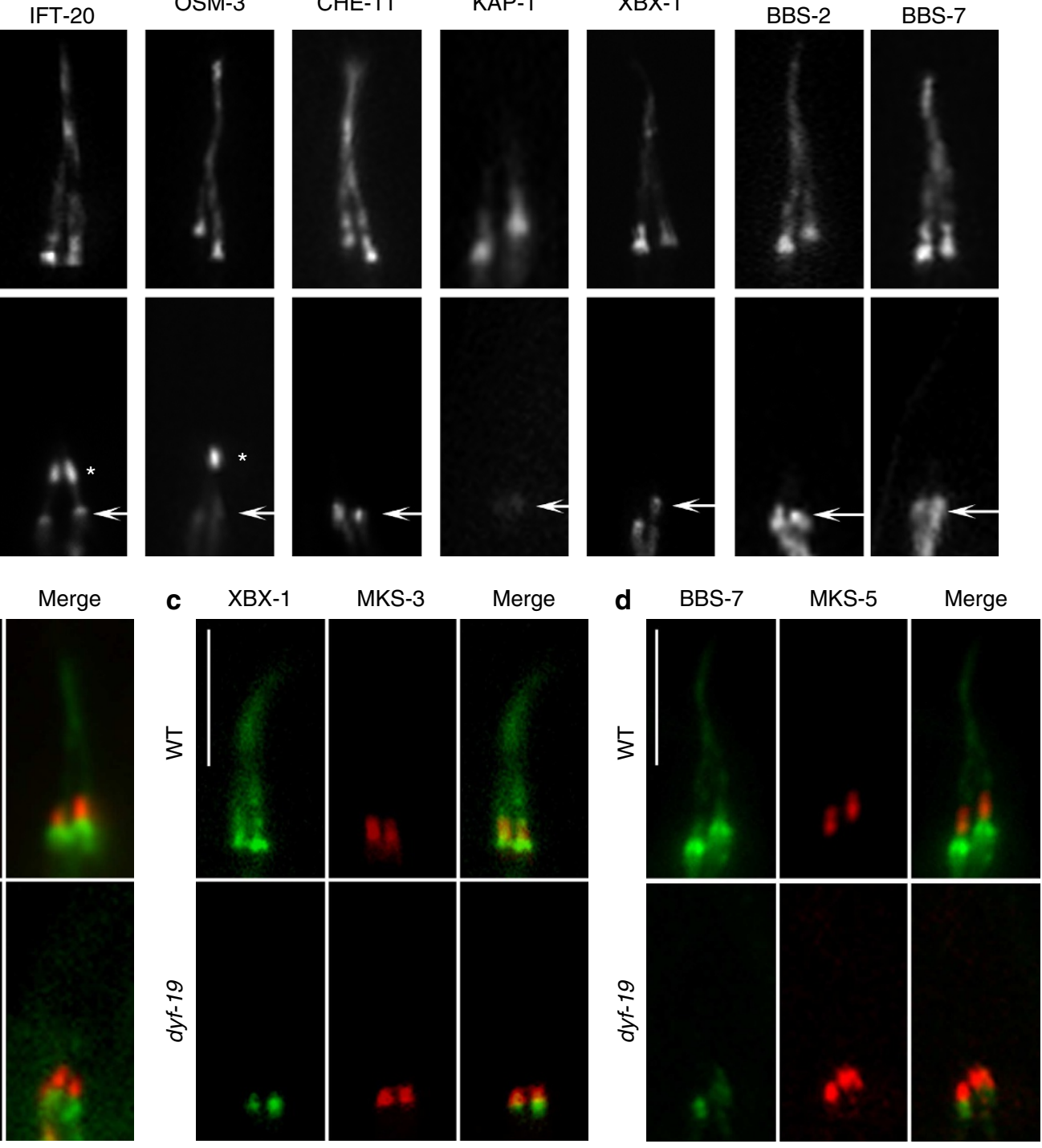

Figure 2 | The ciliary entry of IFT components is compromised in dyf-19 mutants. (a) Fluorescence micrographs of cilia labelled with various IFT markers. In dyf-19, IFT-B components (OSM-5, CHE-2 and IFT-20) and IFT-B-associated kinesin motor OSM-3/KIF17 show consistent ciliary tip accumulation similar to OSM-6 (Fig. 1C). In contrast, IFT-A components CHE-11, IFT-A-associated kinesin-II subunit KAP-1, dynein light chain XBX-1 and BBSome proteins are all excluded from the cilia. Arrows and arrowheads indicate the base and tip of cilia, respectively. Stars note accumulation. Colabelling with TZ markers revealed that CHE-11 (b), XBX-1 (c) and BBS-7 (d) were restricted below the TZ in dyf-19 mutants. Scale bars, $5 \mu \mathrm{m}$.

mutants. Previously, we successfully applied a bimolecular fluorescence complementation (BiFC) assay to examine IFT integrity in live worms ${ }^{47}$. The BiFC assay was developed to directly visualize protein-protein interactions in the same macromolecular complex in their natural environment ${ }^{48}$. In wt cilia, fluorescence complementation was clearly observed between IFT-A component CHE-11 and IFT-B component IFT-20, between BBSome components BBS-7 and BBS-9, as well as between IFT component DYF-2 (the orthologue of human IFT144) and BBSome component BBS-9, indicating the formation of functional IFT particles (Fig. 3b). The following observations indicate that the complemented IFT protein pairs are functional: they showed characteristic IFT movement (Supplementary Fig. S3a); the fluorescence complementation was disrupted in ift mutants (Supplementary Fig. S3b); and they could rescue ciliogenesis defects of corresponding ift or bbs mutants, respectively (Supplementary Fig. S3c). Notably, consistent with previous observation that IFT components concentrate around $\mathrm{TFs}^{23}$, we found that the majority of complemented BiFC signal concentrated at a similar site in wt cilia (Fig. 3b-d), suggesting that the area around TFs is the major pool for assembled IFT particles. In contrast, in $d y f-19$ mutants, fluorescence complementation was completely restricted to below the TZ (Fig. 3c,d), a site comparable to that where IFT-A and BBSome proteins were found in $d y f-19$ mutants (Fig. 2b-d). Furthermore, no IFT movement could be observed for any of the BiFC pairs examined in $d y f-19$ cilia. Taken together, these observations suggest that the assembled IFT particles fail to pass through TFs in DYF-19-deficient cilia. Interestingly, individual IFT-B components appear to enter the cilia independently of DYF-19, probably regulated by an unknown TF functional component or by compensatory mechanisms.

DYF-19 directly interacts with the IFT-B component DYF-11. When we were examining various IFT components in $d y f-19$ 


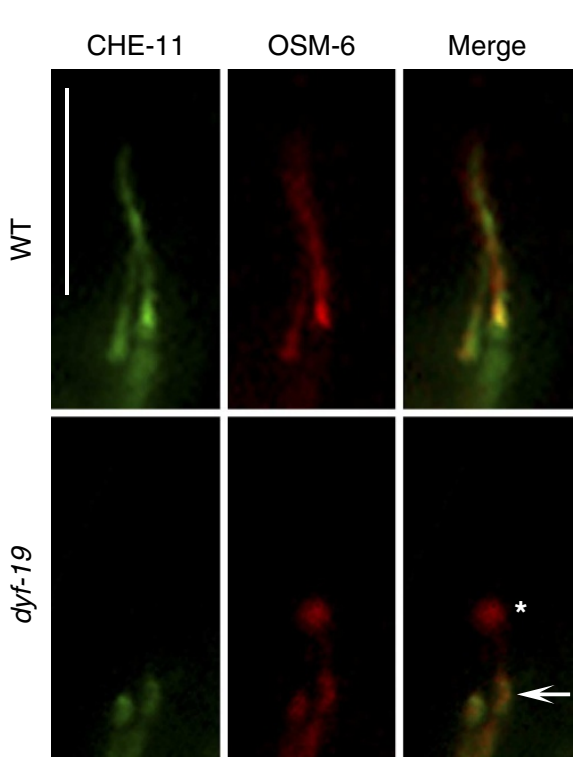

C CHE-11::VN + IFT-20::VC
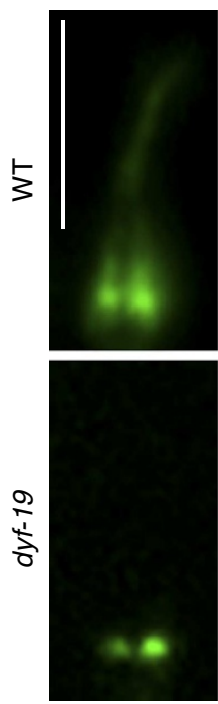

MKS-5
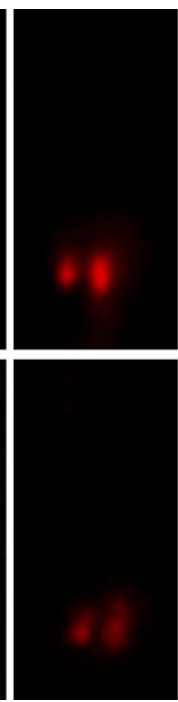

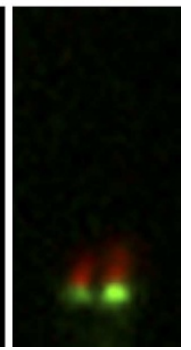

b
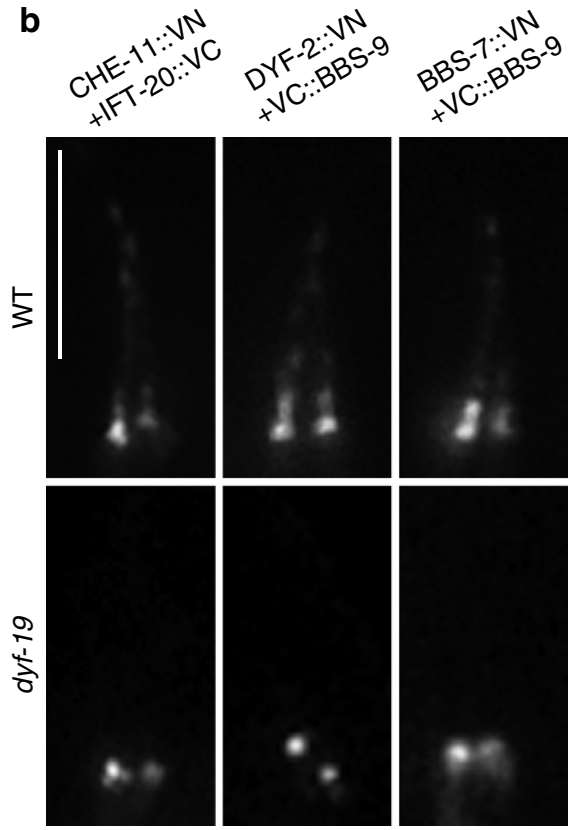

d DYF-2::VN + VC::BBS-9

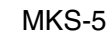

Merge
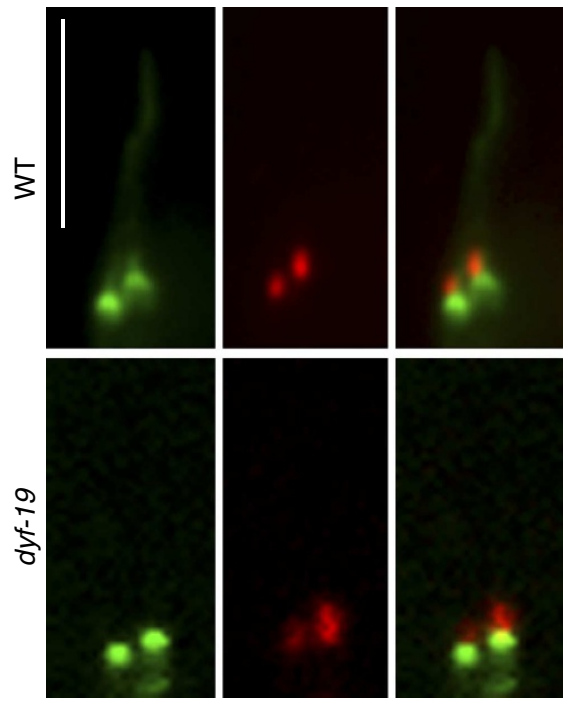

Figure 3 | DYF-19 regulates the ciliary entry of assembled IFT particles. (a) At the base of Wt or dyf-19 cilia, the IFT-A component CHE-11 and the IFT-B component OSM-6 co-localize, presumably below the TZ. (b) In BiFC analyses, fluorescence complementation of CHE-11::VN-IFT-20::VC, DYF-2::VN-VC::BBS-9 and DYF-2::VN-VC::BBS-9 occurs along the entire cilium in Wt, but only at the ciliary base in dyf-19 mutants. Reconstituted CHE-11::VN-IFT-20::VC (c) and DYF-2::VN-VC::BBS-9 (d) localize just below the TZ marker in dyf-19 mutants. VN: N terminus of Venus yellow fluorescent protein (YFP); VC: $C$ terminus of Venus YFP. Bars, $5 \mu \mathrm{m}$.

mutants, DYF-11 (the orthologue of human IFT-B component IFT54) came to our attention. Unlike other IFT-B components (Fig. 2a), small amounts of DYF-11 entered cilia but showed no accumulation, and the majority of DYF-11 was restricted below the TFs (Fig. 4a). This unique mislocalization phenotype was not observed in any other ift mutants (Fig. 4a), leading us to suspect that DYF-11 might be the effector of DYF-19 in regulating the ciliary entry of IFT particles. Indeed, we found that DYF-19 interacts directly with the amino terminus of DYF-11 (Fig. 4b). As expected, we visualized strong fluorescence complementation between DYF-19 and DYF-11 around TFs in the BiFC assay (Fig. 4c). In contrast, we detected no or very weak fluorescence complementation between DYF-19 and other IFT components, either the IFT-A component CHE-11 or the IFT-B component
OSM-6, in wt cilia (Fig. 4c). These observations indicate that DYF-11 is probably the adaptor that bridges the IFT particle to DYF-19 around TFs. Moreover, as reported previously ${ }^{49}$, we noted that $d y f-11$ mutants phenocopy $d y f-19$ mutants in that IFT-B components enter truncated cilia, but IFT-A components fail to do so (Fig. 4d). However, the fact that the CHE-11-IFT-20 BiFC signal was totally disrupted in $d y f-11$ mutants (Supplementary Fig. S3b), probably due to the role of DYF-11 as an essential IFT structural component, prevented us from further examining whether assembled IFT particles fail to enter DYF-11-deficient cilia. Taken together, our results suggest that the TF component DYF-19 regulates the ciliary entry of assembled IFT particles through a direct interaction with the IFT component DYF-11 (Fig. 4e). 


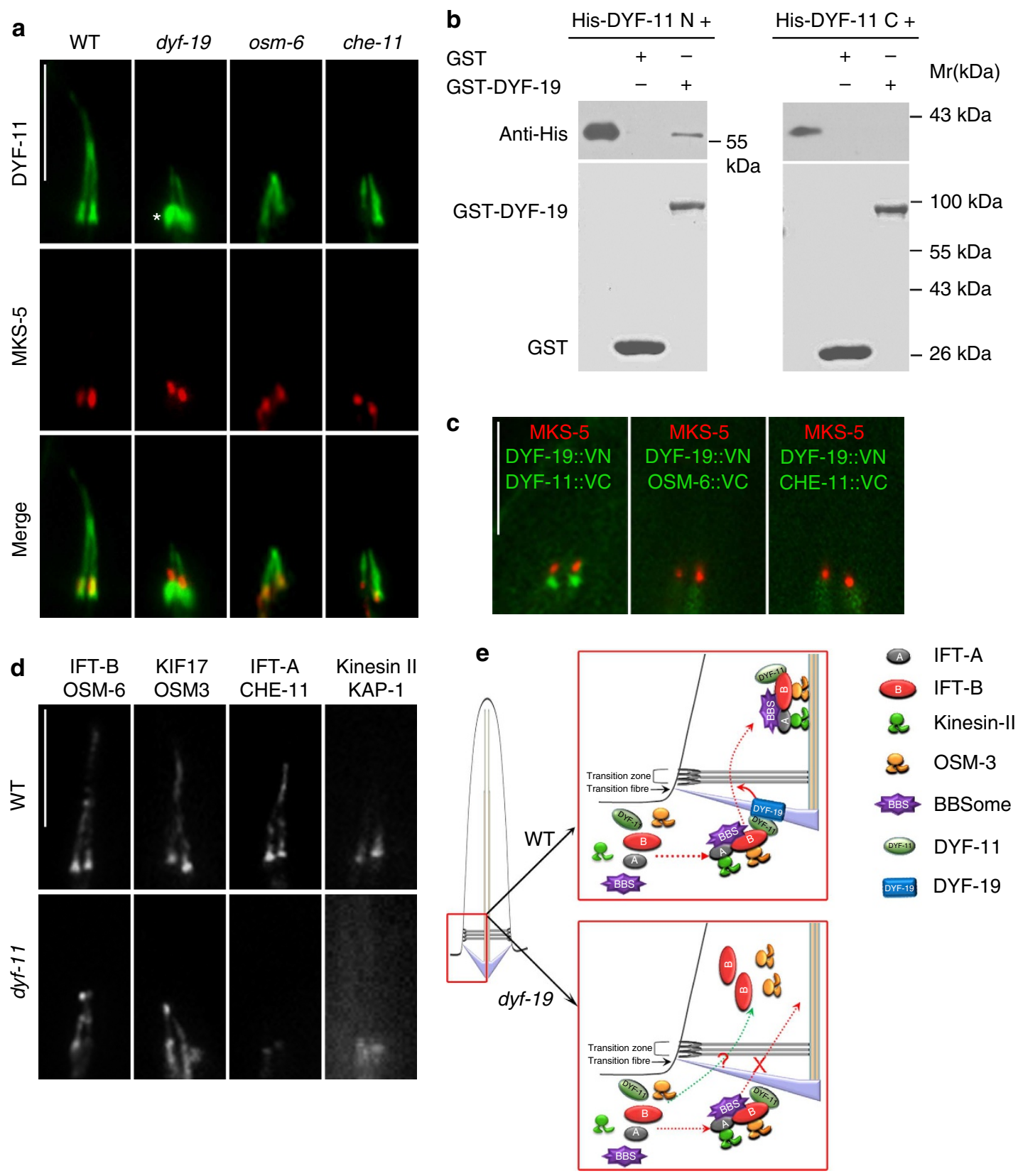

Figure 4 | DYF-19 interacts directly with IFT component DYF-11. (a) DYF-11 shows a unique mislocalization pattern in DYF-19-deficient cilia. (b) The DYF-11 N terminus interacts directly with DYF-19 in a GST pull-down assay. (c) At the ciliary base, stable fluorescence complementation in BiFC assays occurs between DYF-19 and DYF-11 but no or very weak between DYF-19 and other IFT components below the TZ. (d) Dyf-11 mutants show similar phenotypes to dyf-19 mutants for the ciliary entry of IFT components. (e) Schematic of the role of TF component DYF-19 in regulating the ciliary entry of assembled IFT particles through a direct interaction with the IFT component DYF-11. VN: N terminus of Venus yellow fluorescent protein (YFP); VC: $C$ terminus of Venus YFP. Scale bars, $5 \mu \mathrm{m}$.

The localization and function of DYF-19 is highly conserved. As DYF-19 is conserved between worms and humans, we next asked whether its mammalian homologue, FBF1, has a similar role. As expected, both endogenous FBF1 and overexpressed, Flag-tagged FBF1 consistently formed a ring-like structure around the mother centriole or at the ciliary base in IMCD3 cells (Fig. 5a-c). On mother centrioles, FBF1 co-localized with the DAP marker CEP164 (ref. 24) and localized above the subDAP marker ODF2 (Fig. 5d). Immuno-electron microscopy analyses confirmed that FBF1 specifically localized along distal appendages of the mother centriole (Fig. 5e). Distal appendages exist only on mother centrioles. Consistently, FBF1 was only restricted to the mother centriole during G1/S and G2 phases of the cell cycle. At the late G2 phase or early M phase, FBF1 started to appear on the other maturing centriole, and the signal increased during subsequent stages of mitosis (Supplementary Fig. S4).

Depletion of FBF1 resulted in severely truncated cilia (Fig. 5f-h). Similar to what we observed in C. elegans DYF-19-deficient cilia, the IFT-B component IFT88 but not the IFT-A component IFT140 could enter the truncated cilia (Fig. 5i,j). As expected, we detected a direct interaction between FBF1 and IFT54, the mouse orthologue of worm DYF-11, in a glutathione S-transferase (GST) pull-down assay (Supplementary Figs S5). 
a

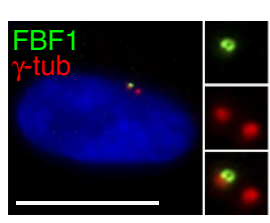

b

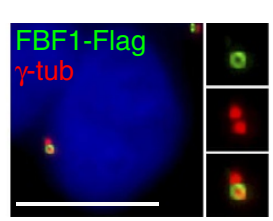

d

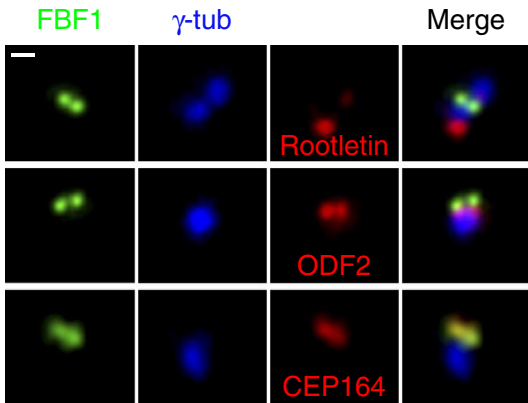

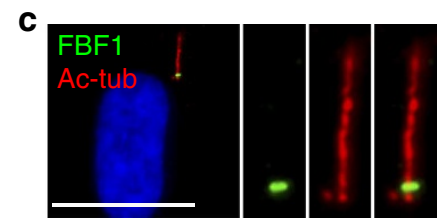

e
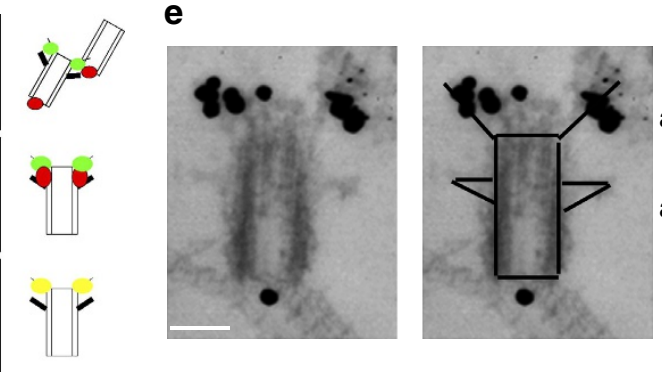

Distal

appendages

Subdistal

appendages
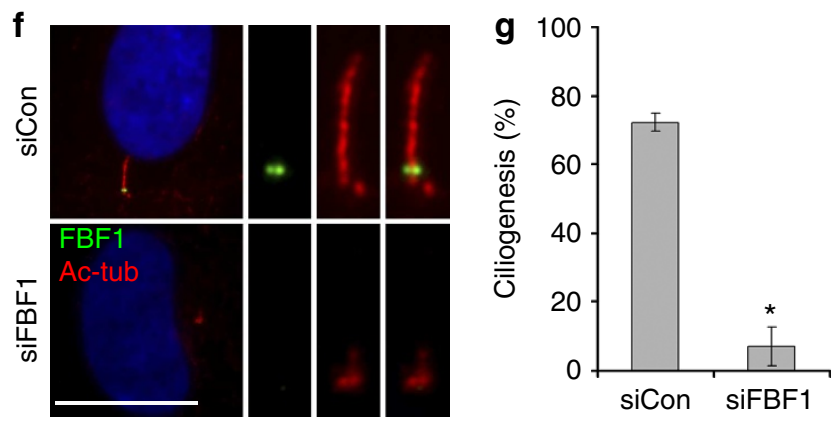

h
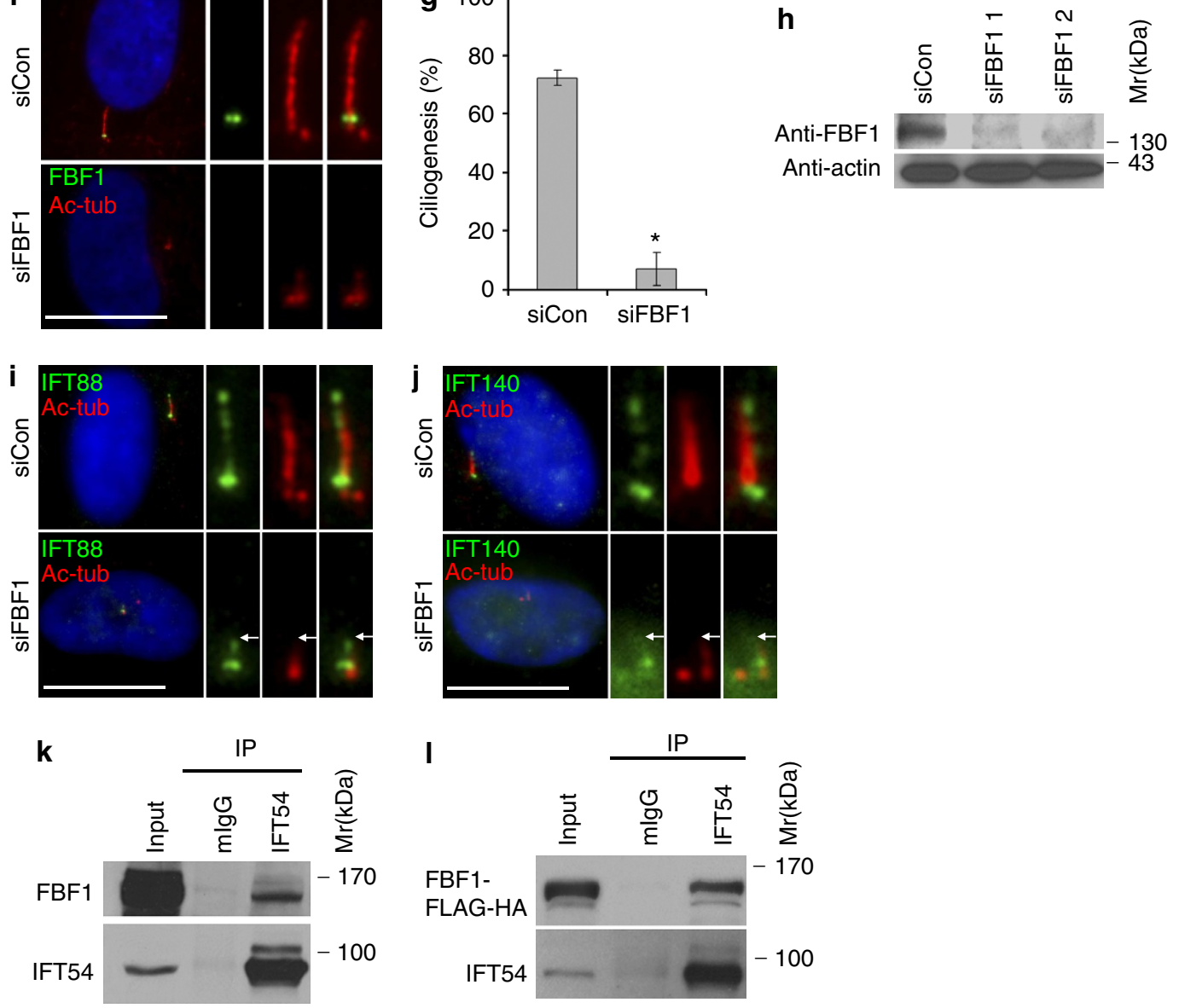

Figure 5 | The localization and function of DYF-19 are highly conserved. Either endogenous (a) or overexpressed (b) FBF1, the mammalian homologue of worm DYF-19, localizes specifically on one centriole with a ring-like pattern in IMCD3 cells. (c) FBF1 localizes at the ciliary base, above the basal body. (d) In IMCD3 cells, FBF1 localizes above rootlet and sub-DAP ODF2 and completely co-localizes with DAP CEP164. (e) Immuno-electron microscopy demonstrates that FBF1 localizes specifically to distal appendages of mother centrioles. (f-h) Knockdown of FBF1 leads to severely truncated cilia in most RNA interference (RNAi)-treated hTERT-RPE cells. Data are represented as mean of three independent experiments $(n=200)$ and error bars indicate s.d. Significant differences were identified by the Student's $t$-test. ${ }^{*} P<0.001$. (i,j) The IFT-B component IFT88, but not the IFT-A component IFT140, enters the truncated cilia of FBF1 knockdown hTERT-RPE cells. Arrows indicate the tips of truncated cilia. (k) Endogenous IFT54 immunoprecipitates with FBF1 in HEK293 cells. (I) HEK293 cells were transiently transfected with FLAG-HA-tagged FBF1, and $48 \mathrm{~h}$ later cells were subjected to immunoprecipitation using normal mouse IgG (mlgG) or anti-IFT54 antibody. Fifty micrograms of protein were loaded into each lane. Scale bars, (d) $1 \mu \mathrm{m}$; (e) $200 \mathrm{~nm}$; others, $20 \mu \mathrm{m}$.

Immunoprecipitation confirmed that endogenous IFT54 associates with either endogenous FBF1 or overexpressed FBF1 (Fig. 5k,l). Further mapping of the interaction indicated that the IFT54
$\mathrm{N}$ terminus (aa 1-325) probably interacts with FBF1 at multiple sites, but with a stronger binding at $\mathrm{FBF} 1$ carboxy terminus (aa 816-1,075; Supplementary Fig. S5). 


\section{Discussion}

In summary, FBF1 (or DYF-19) specifically targets to TFs, directly interacts with the IFT component IFT54 (or DYF-11) and has a role in regulating the passage of assembled IFT particles into the cilium. The highly conserved subcellular localization and function across species strongly support the conclusion that FBF1 (or DYF-19) is a core functional determinant that makes TFs a key site for regulating the ciliary import of assembled IFT machinery.

Since the discovery of the cilium more than a century ago, a central question in cilia biology is how the sensory cilium is functionally separated from the cytosol. At the ciliary base, there is a size-dependent diffusion barrier that restricts the ciliary entry of large proteins ${ }^{11,12}$, indicating that a passive gate selectively regulates the ciliary entry of various ciliogenic proteins. The size of most IFT components is larger than $60 \mathrm{kDa}$ and the assembled IFT particle is larger than $1 \mathrm{MDa}$. How the IFT particle or its components actively pass through the ciliary gate remains a mystery. The obvious pore complex formed by Y-links of the TZ at the ciliary base led to the notion that the Y-links probably act as the active ciliary gate ${ }^{7,50}$. However, the depletion of $\mathrm{TZ}$ components causes no major defect in the ciliary entry of IFT particles or of any IFT components ${ }^{20}$, indicating that an active gating mechanism functions upstream of the TZ. Our observations suggest that TFs, which form a cartwheel structure just below the TZ, probably form the ciliary gate for IFT machinery, and FBF1 (or DYF-19) actively facilitates the passage of assembled IFT particles through TFs.

It is likely to be that FBF1 (DYF-19) provides a docking site on TFs for assembled IFT particles by its direct interaction with the IFT component IFT54 (DYF-11). After docking of IFT particles, the ciliary pore complex (CPC), a proposed nuclear pore complex (NPC)-like structure ${ }^{11,51-53}$, might remodel and facilitate the import of assembled IFT particles. Many interesting questions still remain: does FBF1 (DYF-19) and/or TFs determine the ciliary entry of other ciliogenic proteins, such as sensory receptors and signalling molecules? What is the identity of the redundant $\mathrm{TF}$ component that regulates the ciliary entry of IFT-B components in DYF-19-deficient cilia? What is the correlation between TF malfunction and ciliopathies? The molecular exploration of TFs is still in its infancy. Current and future studies of the FBF1 (DYF-19) pathway will not only reveal the molecular identity of TFs but also contribute significantly to our understanding of how TFs define the cilium as a functionally and structurally distinct sensory organelle.

\begin{abstract}
Methods
Strains. Strains used in this study are listed in Supplementary Table S1. Standard conditions were used to culture and maintain worms. Standard genetic crosses were used to introduce reporter transgenes from wt worms into mutant worms. Jhu455 and jhu546 mutants were isolated during an EMS screening for Dyf mutants. Standard single-nucleotide polymorphism mapping was used to clone the mutation. Nonsense mutations at nt880 and nt1219 of the Y43F8C.4 coding region were discovered in $j h u 455$ and jhu546, respectively. Before phenotypic analyses and the introduction of markers, mutants were outcrossed six times against the $\mathrm{N} 2 \mathrm{wt}$
\end{abstract}

Antibodies and reagents. Primary antibodies used in this study include the following: mouse monoclonal antibodies against Odf2 (1A1, Novus, H00004957M01, 1:400), acetylated tubulin (6-11B-1, Sigma, T7451, 1:400), $\gamma$-tubulin (GTU-88, Sigma, T5326, 1:400), polyglutamylated tubulin (GT335, AdipoGen, AG-20B-0020-C100, 1:400), Flag (clone M2, Sigma, F1804, 1:1,000), His (clone HIS.H8, Millipore, 1:1,000) and actin (AC-15, Sigma, A1978, 1:1,000); rabbit polyclonal antibodies against Cep164 (Sigma, SAB3500022, 1:400), FBF1 (Proteintech, 11531-1-AP, 1:400), IFT88 (Proteintech, 13967-1-AP, 1:400) and IFT140 (Proteintech, 1740-1-AP, 1:400); mouse polyclonal antibody against IFT54 (Abcam ab68958, 1:400); and goat polyclonal antibody against Rootletin (C-20, Santa Cruz, sc-67824, 1:400). All secondary antibodies (goat anti-mouse, rabbit or donkey anti-mouse and rabbit or goat conjugated with Alex Fluor 488, 555, or 647) were purchased from Invitrogen.
Statistics. Significant differences were identified by the Student's $t$-test $\left({ }^{*} P<0.001\right)$. Error bars indicate s.d. For the worm study, $n=300$ animals; five independent experiments were performed. For the cell study, $n=200$ cells; three independent experiments were performed.

EMS mutagenesis screen and dye-filling assay. N2 animals were mutagenized with EMS using standard procedures. F2 progeny from mutagenized animals were grown at $20^{\circ} \mathrm{C}$, and adult animals were incubated in diluted DiI dye (Molecular Probes, 1:200 dilution in M9 of the $2 \mathrm{mg} \mathrm{ml}^{-1}$ stock in dimethyl formamide) for $1 \mathrm{~h}$ at room temperature. After incubation, the animals were washed at least three times with M9 and observed using a fluorescence microscope (M2Bio, Zeiss). Worms that showed defective dye filling were selected.

Microscopy and imaging. Live, healthy adult worms were anaesthetized in $10 \mathrm{mM}$ levamisole and mounted on $5 \%$ agarose pads. Images were acquired using either an imaging microscope (Nikon TE 2000-U) with a Plan Apochromat $60 \times 1.49$ oilimmersion objective (Nikon) or a Zeiss LSM 780 confocal laser-scanning microscope equipped with a Plan Apochromat $\times 100$ oil-immersion objective. Motility stacks were recorded using a charge-coupled device camera (Photometrics QuantEM 512SC, Roper Scientific) and kymographs were produced with the Metamorph software.

Transmission electron microscopy. Transmission electron microscopy on worm amphid cilia was performed as described previously ${ }^{54}$. Briefly, worms were washed and then fixed in $2.5 \%$ glutaraldehyde in cacodylate buffer. To facilitate fixation, worm heads were cut off using a syringe needle. Animals were rinsed and then post fixed with $1 \%$ osmium tetroxide in cacodylate buffer for $1 \mathrm{~h}$ at $4{ }^{\circ} \mathrm{C}$. Worm heads were oriented and embedded in agarose, and then dehydrated and embedded in Embed 812 resin according to standard procedures ${ }^{55}$. Thin sections from the anterior tip of the worm head were collected on a diamond knife and post stained before viewing on an electron microscope (JEM-1400, JEOL).

Immuno-electron microscopy. For pre-embedding immuno-electron microscopy, centrosomes were isolated from U2OS cells, fixed with $4 \%$ formaldehyde for 20 min and permeabilized with PBS $+0.1 \%$ Triton X-100 for 30 min. Blocking and primary antibody incubations were then performed, followed by incubation with goat anti-rabbit IgG-Nanogold. Nanogold was silver enhanced with HQ Silver (Nanoprobes) and samples were further processed using standard methods by the EM core facility at Mayo Clinic, Rochester.

Cell culture and small interfering RNA. Human telomerase-immortalized retinal-pigmented epithelial cells (hTERT-RPE-1) and mouse inner medullar collecting duct cells (IMCD3) were cultured in DMEM/F12 supplemented with $10 \%$ fetal bovine serum. HEK293 cells were grown in DMEM with 10\% fetal bovine serum. To induce cilia formation, cells were starved for $24-48 \mathrm{~h}$ in media without serum after they were grown to confluence.

Mouse Fbf1 (NM_172571) was used for all subcloning purposes. FLAG-HA double-tagged FBF1 plasmids were transfected into cells using Lipofectamine 2000 (Invitrogen). To obtain RPE or IMCD3 clones stably expressing FBF1 in the centrosome, single-cell clones with a lower expression level were selected.

To knock down FBF1, RPE cells were transfected with $50 \mathrm{nM}$ small interfering (siRNA) using Lipofectamine RNAiMAX (Invitrogen) following the manufacturer's instructions. Two consecutive siRNA transfections at a 72-h interval were performed. siRNA sequences targeting human Fbfl ( $5^{\prime}$-TGAACAGTTCTTCCT GGAG- $3^{\prime}$ and $5^{\prime}$-CCGAGGAGGTGGAGAGCAT- $3^{\prime}$ ) were used.

Immunofluorescence. For centrosome staining, cells were fixed in $-20^{\circ} \mathrm{C}$ methanol for $20 \mathrm{~min}$, then blocked in $3 \%$ BSA and sequentially incubated with primary and secondary antibodies.

For cilia staining, cells were typically fixed with $4 \%$ paraformaldehyde for $20 \mathrm{~min}$ at room temperature, followed by permeabilization with $0.2 \%$ Triton X-100 for $10 \mathrm{~min}$. Cells were then blocked in 3\% BSA and sequentially incubated with primary and secondary antibodies.

To stain cilia using the IFT140 antibody, we used an enhanced immunofluorescence protocol ${ }^{56}$. Cells were prefixed with $0.4 \%$ paraformaldehyde for $5 \mathrm{~min}$ at $37^{\circ} \mathrm{C}$, extracted with $0.5 \%$ Triton X-100 in PHEM (50 mM PIPES, $50 \mathrm{mM}$ HEPES, $10 \mathrm{mM}$ EGTA and $10 \mathrm{mM} \mathrm{MgCl} 2, \mathrm{pH}$ 6.9) for $2 \mathrm{~min}$ at $37^{\circ} \mathrm{C}$ and then stained following the immunofluorescence procedure described above.

Immunoprecipitation and GST pull-down assay. Immunoprecipitation and GST pull-down assay were performed as described previously ${ }^{57}$. Briefly, HEK293 cells or HEK293 cells expressing double-tagged FLAG-HA FBF1 were lysed in lysis buffer (25 mM Tris-HCl, pH 7.4, $150 \mathrm{mM} \mathrm{NaCl}, 0.5 \%$ NP-40, $1 \mathrm{mM}$ EDTA and protease inhibitors). The lysate was centrifuged for $20 \mathrm{~min}$ at $12000 \mathrm{~g}$ at $4{ }^{\circ} \mathrm{C}$ and then the supernatant was precleared with protein G Sepharose beads for $4 \mathrm{~h}$. After removal of protein $\mathrm{G}$, the supernatant was incubated with protein $\mathrm{G}$ beads and $2 \mu \mathrm{g}$ of either anti-IFT54 (Abcam) or mouse IgG (Santa Cruz) overnight at $4{ }^{\circ} \mathrm{C}$. 
After washing the beads five times, the immunocomplexes were separated by SDSPAGE gels and analysed.

For GST pull-down assays, pET28a and pGEX-4 T-1 constructs were transformed into BL21 (DE3) (Novagen). Proteins were expressed and purified using His resin or glutathione sepharose. Purified His-DYF-11, His-IFT54 or their truncations were incubated with GST, GST-DYF-9, GST-FBF1 or their truncations immobilized on glutathione sepharose in the binding buffer $(25 \mathrm{mM}$ Tris- $\mathrm{HCl}, \mathrm{pH}$ $7.4,150 \mathrm{mM} \mathrm{NaCl}, 0.5 \%$ Triton X-100, $1 \mathrm{mM}$ dithiothreitol, $10 \%$ glycerol and protease inhibitors) for $4 \mathrm{~h}$ at $4{ }^{\circ} \mathrm{C}$. The beads were then washed five times with binding buffer and analysed by western blotting. Full scans of western blots are provided in Supplementary Fig. S8.

BiFC assay. The BiFC assay was used to directly visualize the IFT-A, IFT-B and BBSome complexes in living worms ${ }^{47}$. The following combinations were used in this study: the IFT-A component CHE-11::VN173 with the IFT-B component IFT20::VC155; the IFT component VC155::DYF-2 with the BBSome component VN173::BBS-9; and the BBSome components VN173::BBS-9 and VC::BBS-7. To examine the BiFC signals relative to the $\mathrm{TZ}$, plasmids in these combinations were co-injected along with the TZ marker MKS-5::mCherry and the co-injection marker pRF4 (rol-6(su1006)) into wt worms $\left(5 \mathrm{ng} \mu \mathrm{l}^{-1}\right.$ for each BiFC plasmid and MKS-5 vector, $\left.100 \mathrm{ng}^{-1} \mathrm{l}^{-1} \mathrm{pRF} 4\right)$. Once a stable line was obtained, the same transgene was crossed into various mutants. Fluorescent signals were visualized by using the yellow fluorescent protein filter.

\section{References}

1. Singla, V. \& Reiter, J. F. The primary cilium as the cell's antenna: signaling at a sensory organelle. Science 313, 629-633 (2006).

2. Goetz, S. C. \& Anderson, K. V. The primary cilium: a signalling centre during vertebrate development. Nat. Rev. Genet. 11, 331-344 (2010).

3. Hildebrandt, F., Benzing, T. \& Katsanis, N. Ciliopathies. N. Engl. J. Med. 364, 1533-1543 (2011)

4. Badano, J. L., Mitsuma, N., Beales, P. L. \& Katsanis, N. The ciliopathies: an emerging class of human genetic disorders. Annu. Rev. Genomics Hum. Genet. 7, 125-148 (2006).

5. Hao, L. \& Scholey, J. M. Intraflagellar transport at a glance. J. Cell Sci. 122, 889-892 (2009).

6. Pedersen, L. B. \& Rosenbaum, J. L. Intraflagellar transport (IFT) role in ciliary assembly, resorption and signalling. Curr. Top. Dev. Biol. 85, 23-61 (2008).

7. Rosenbaum, J. L. \& Witman, G. B. Intraflagellar transport. Nat. Rev. Mol. Cell Biol. 3, 813-825 (2002).

8. Ishikawa, H. \& Marshall, W. F. Ciliogenesis: building the cell's antenna. Nat. Rev. Mol. Cell Biol. 12, 222-234 (2011).

9. Qin, H., Diener, D. R., Geimer, S., Cole, D. G. \& Rosenbaum, J. L. Intraflagellar transport (IFT) cargo: IFT transports flagellar precursors to the tip and turnover products to the cell body. J. Cell Biol. 164, 255-266 (2004).

10. Fowkes, M. E. \& Mitchell, D. R. The role of preassembled cytoplasmic complexes in assembly of flagellar dynein subunits. Mol. Biol. Cell 9, 2337-2347 (1998)

11. Kee, H. L. et al. A size-exclusion permeability barrier and nucleoporins characterize a ciliary pore complex that regulates transport into cilia. Nat. Cell. Biol. 14, 431-437 (2012).

12. Lin, Y. C. et al. Chemically inducible diffusion trap at cilia reveals molecular sieve-like barrier. Nat. Chem. Biol. 9, 437-443 (2013).

13. Reiter, J. F., Blacque, O. E. \& Leroux, M. R. The base of the cilium: roles for transition fibres and the transition zone in ciliary formation, maintenance and compartmentalization. EMBO Rep. 13, 608-618 (2012).

14. Anderson, R. G. The three-dimensional structure of the basal body from the rhesus monkey oviduct. J. Cell Biol. 54, 246-265 (1972).

15. Gilula, N. B. \& Satir, P. The ciliary necklace. A ciliary membrane specialization. J. Cell Biol. 53, 494-509 (1972).

16. Czarnecki, P. G. \& Shah, J. V. The ciliary transition zone: from morphology and molecules to medicine. Trends Cell Biol. 22, 201-210 (2012).

17. Sang, L. et al. Mapping the NPHP-JBTS-MKS protein network reveals ciliopathy disease genes and pathways. Cell 145, 513-528 (2011).

18. Chih, B. et al. A ciliopathy complex at the transition zone protects the cilia as a privileged membrane domain. Nat. Cell Biol. 14, 61-72 (2012).

19. Garcia-Gonzalo, F. R. et al. A transition zone complex regulates mammalian ciliogenesis and ciliary membrane composition. Nat. Genet. 43, 776-784 (2011).

20. Williams, C. L. et al. MKS and NPHP modules cooperate to establish basal body/transition zone membrane associations and ciliary gate function during ciliogenesis. J. Cell Biol. 192, 1023-1041 (2011).

21. Craige, B. et al. CEP290 tethers flagellar transition zone microtubules to the membrane and regulates flagellar protein content. J. Cell Biol. 190, 927-940 (2010).

22. Jauregui, A. R., Nguyen, K. C., Hall, D. H. \& Barr, M. M. The Caenorhabditis elegans nephrocystins act as global modifiers of cilium structure. J. Cell Biol. 180, 973-988 (2008).
23. Deane, J. A., Cole, D. G., Seeley, E. S., Diener, D. R. \& Rosenbaum, J. L. Localization of intraflagellar transport protein IFT52 identifies basal body transitional fibres as the docking site for IFT particles. Curr. Biol. 11, 1586-1590 (2001).

24. Graser, S. et al. Cep164, a novel centriole appendage protein required for primary cilium formation. J. Cell Biol. 179, 321-330 (2007).

25. Tanos, B. E. et al. Centriole distal appendages promote membrane docking, leading to cilia initiation. Genes Dev. 27, 163-168 (2013).

26. Joo, K. et al. CCDC41 is required for ciliary vesicle docking to the mother centriole. Proc. Natl Acad. Sci. USA 110, 5987-5992 (2013).

27. Schmidt, K. N. et al. Cep164 mediates vesicular docking to the mother centriole during early steps of ciliogenesis. J. Cell Biol. 199, 1083-1101 (2012).

28. Hedgecock, E. M., Culotti, J. G., Thomson, J. N. \& Perkins, L. A. Axonal guidance mutants of Caenorhabditis elegans identified by filling sensory neurons with fluorescein dyes. Dev. Biol. 111, 158-170 (1985).

29. Schmidt, T. et al. A novel protein (Fbf-1) that binds to CD95/APO-1/FAS and shows sequence similarity to trichohyalin and plectin. Biochim. Biophys. Acta 1493, 249-254 (2000).

30. Sugimoto, M. et al. The keratin-binding protein Albatross regulates polarization of epithelial cells. J. Cell Biol. 183, 19-28 (2008).

31. Jakobsen, L. et al. Novel asymmetrically localizing components of human centrosomes identified by complementary proteomics methods. EMBO J. 30, 1520-1535 (2011).

32. Kaplan, O. I. et al. Endocytosis genes facilitate protein and membrane transport in C. elegans sensory cilia. Curr. Biol. 22, 451-460 (2012).

33. Williams, C. L., Winkelbauer, M. E., Schafer, J. C., Michaud, E. J. \& Yoder, B. K. Functional redundancy of the B9 proteins and nephrocystins in Caenorhabditis elegans ciliogenesis. Mol. Biol. Cell 19, 2154-2168 (2008).

34. Williams, C. L., Masyukova, S. V. \& Yoder, B. K. Normal ciliogenesis requires synergy between the cystic kidney disease genes MKS-3 and NPHP-4. J. Am. Soc. Nephrol. 21, 782-793 (2010).

35. Warburton-Pitt, S. R. et al. Ciliogenesis in Caenorhabditis elegans requires genetic interactions between ciliary middle segment localized NPHP-2 (inversin) and transition zone-associated proteins. J. Cell Sci. 125, 2592-2603 (2012).

36. Pazour, G. J., Wilkerson, C. G. \& Witman, G. B. A dynein light chain is essential for the retrograde particle movement of intraflagellar transport (IFT) J. Cell Biol. 141, 979-992 (1998).

37. Pazour, G. J., Dickert, B. L. \& Witman, G. B. The DHC1b (DHC2) isoform of cytoplasmic dynein is required for flagellar assembly. J. Cell Biol. 144, 473-481 (1999).

38. Signor, D. et al. Role of a class DHC1b dynein in retrograde transport of IFT motors and IFT raft particles along cilia, but not dendrites, in chemosensory neurons of living Caenorhabditis elegans. J. Cell Biol. 147, 519-530 (1999).

39. Wicks, S. R., de Vries, C. J., van Luenen, H. G. \& Plasterk, R. H. CHE-3, a cytosolic dynein heavy chain, is required for sensory cilia structure and function in Caenorhabditis elegans. Dev. Biol. 221, 295-307 (2000).

40. Piperno, G. et al. Distinct mutants of retrograde intraflagellar transport (IFT) share similar morphological and molecular defects. J. Cell Biol. 143, 1591-1601 (1998).

41. Schafer, J. C., Haycraft, C. J., Thomas, J. H., Yoder, B. K. \& Swoboda, P. XBX-1 encodes a dynein light intermediate chain required for retrograde intraflagellar transport and cilia assembly in Caenorhabditis elegans. Mol. Biol. Cell 14, 2057-2070 (2003).

42. Loktev, A. V. et al. A BBSome subunit links ciliogenesis, microtubule stability, and acetylation. Dev. Cell 15, 854-865 (2008).

43. Nachury, M. V. et al. A core complex of BBS proteins cooperates with the GTPase Rab8 to promote ciliary membrane biogenesis. Cell 129, 1201-1213 (2007).

44. Lechtreck, K. F. et al. Cycling of the signaling protein phospholipase D through cilia requires the BBSome only for the export phase. J. Cell Biol. 201, 249-261 (2013).

45. Lechtreck, K. F. et al. The Chlamydomonas reinhardtii BBSome is an IFT cargo required for export of specific signaling proteins from flagella. J. Cell Biol. 187, 1117-1132 (2009).

46. Ou, G., Blacque, O. E., Snow, J. J., Leroux, M. R. \& Scholey, J. M. Functional coordination of intraflagellar transport motors. Nature 436, 583-587 (2005).

47. Wei, Q. et al. The BBSome controls IFT assembly and turnaround in cilia. Nat. Cell Biol. 14, 950-957 (2012).

48. Hu, C. D., Chinenov, Y. \& Kerppola, T. K. Visualization of interactions among bZIP and Rel family proteins in living cells using bimolecular fluorescence complementation. Mol. Cell 9, 789-798 (2002).

49. Li, C. et al. An essential role for DYF-11/MIP-T3 in assembling functional intraflagellar transport complexes. PLoS Genet. 4, e1000044 (2008).

50. Satir, P. \& Christensen, S. T. Overview of structure and function of mammalian cilia. Annu. Rev. Physiol. 69, 377-400 (2007).

51. Dishinger, J. F. et al. Ciliary entry of the kinesin-2 motor KIF17 is regulated by importin-beta2 and RanGTP. Nat. Cell Biol. 12, 703-710 (2010). 
52. Hurd, T. W., Fan, S. \& Margolis, B. L. Localization of retinitis pigmentosa 2 to cilia is regulated by Importin beta2. J. Cell Sci. 124, 718-726 (2011).

53. Ounjai, P. et al. Architectural insights into a ciliary partition. Curr. Biol. 23, 339-344 (2013).

54. Li, Y., Wei, Q., Zhang, Y., Ling, K. \& Hu, J. The small GTPases ARL-13 and ARL-3 coordinate intraflagellar transport and ciliogenesis. J. Cell Biol. 189, 1039-1051 (2010).

55. Hall, D. H. Electron microscopy and three-dimensional image reconstruction. Methods Cell Biol. 48, 395-436 (1995).

56. Jin, H. et al. The conserved Bardet-Biedl syndrome proteins assemble a coat that traffics membrane proteins to cilia. Cell 141, 1208-1219 (2010).

57. Li, Y., Zhang, Q., Wei, Q., Zhang, Y., Ling, K. \& Hu, J. SUMOylation of the small GTPase ARL-13 promotes ciliary targeting of sensory receptors. J. Cell Biol. 199, 589-598 (2012).

\section{Acknowledgements}

We thank the Caenorhabditis Genetics Center, the Japanese Bioresource Project and Drs Maureen Barr, Jonathan Scholey, Bradley Yoder and Michel Leroux for strains. J.H. and co-workers were supported by the National Institutes of Health research grant 1R01DK090038 and P30 centre grant P30DK90728, a Pilot and Feasibility Award from the Mayo Clinic Center for Cell Signaling in Gastroenterology (P30DK084567) and the PKD Foundation Young Investigator Award 04YI09a. K.L. was supported by grants from the National Cancer Institute (NCI; 1R01CA149039-01A1) and Susan G. Komen for the Cure (KG100902).

\section{Author contributions}

J.H. and K.L. conceived the project. J.H., Q.W. and Y.L. performed the screening and Q.W. mapped the mutants. Q.W. performed most of the experiments; Q.X. performed the immuno-electron microscopy experiment; Y.Z., Q.Z. and Z.H. generated transgenic animals. K.L. and J.H. wrote the manuscript with contribution of Q.W. P.C.H. and V.E.T. contributed reagents and materials, and discussed the manuscript.

\section{Additional information}

Supplementary Information accompanies this paper at http://www.nature.com/ naturecommunications

Competing financial interests: The authors declare no competing financial interests.

Reprints and permission information is available online at http://npg.nature.com/ reprintsandpermissions/

How to cite this article: Wei, Q. et al. Transition fibre protein FBF1 is required for the ciliary entry of assembled intraflagellar transport complexes. Nat. Commun. 4:2750 doi: $10.1038 /$ ncomms3750 (2013). 